\title{
Observation of Extreme Sea Waves in a Space-Time Ensemble
}

\author{
Alvise BenETAZZO AND FrANCESCO BARBARIOL \\ Institute of Marine Sciences-Italian National Research Council (ISMAR-CNR), Venice, Italy \\ FILIPPO BERGAMASCO AND ANDREA TORSELlO \\ DAIS, Università Ca' Foscari, Venice, Italy \\ SANDRO CARNIEL AND MAURO SClAVO \\ Institute of Marine Sciences-Italian National Research Council (ISMAR-CNR), Venice, Italy
}

(Manuscript received 22 January 2015, in final form 28 May 2015)

\begin{abstract}
In this paper, an observational space-time ensemble of sea surface elevations is investigated in search of the highest waves of the sea state. Wave data were gathered by means of a stereo camera system, which was installed on top of a fixed oceanographic platform located in the Adriatic Sea (Italy). Waves were measured during a mature sea state with an average wind speed of $11 \mathrm{~m} \mathrm{~s}^{-1}$. By examining the space-time ensemble, the $3 \mathrm{D}$ wave groups have been isolated while evolving in the $2 \mathrm{D}$ space and grabbed "when and where" they have been close to the apex of their development, thus exhibiting large surface displacements. The authors have selected the groups displaying maximal crest height exceeding the threshold adopted to define rogue waves in a time record, that is, 1.25 times the significant wave height $\left(H_{s}\right)$. The records at the spatial positions where such large crests occurred have been analyzed to derive the empirical distributions of crest and wave heights, which have been compared against standard statistical linear and nonlinear models. Here, the maximal observed wave crests have resulted to be outliers of the standard statistics, behaving as isolated members of the sample, apparently uncorrelated with other waves of the record. However, this study has found that these unexpectedly large wave crests are better approximated by a space-time model for extreme crest heights. The space-time model performance has been improved, deriving a second-order approximation of the linear model, which has provided a fair agreement with the empirical maxima. The present investigation suggests that very large waves may be more numerous than generally expected.
\end{abstract}

\section{Introduction}

In situ observation of waves is a challenging task; apart from the measurement of usual sea state parameters (e.g., the significant wave height), large wave elevations are often missed by classical instrumentation. Indeed, most of the operational wave measurements rely on surfacefollowing buoys, which tend in general to underestimate the displacement of very high waves (Krogstad and Barstow 2000). In addition to this, pointlike observatory systems (buoys, wave probes, etc.) underestimate (especially in short-crested seas) the actual maximum wave

Corresponding author address: Alvise Benetazzo, Institute of Marine Sciences (ISMAR)-Italian National Research Council (CNR), Arsenale Tesa 104, Castello 2737/F, 30122, Venice, Italy. E-mail: alvise.benetazzo@ve.ismar.cnr.it displacements that can occur on sea surface areas even smaller than the wave characteristics dimensions, namely, the wavelength and the crest length (Fedele et al. 2013; Forristall 2011). In recent years, a great effort has therefore been devoted to develop instrumentation apt to capture the complete dynamics of the waves as they propagate both in time and space. In this context, the field of wave observation has lately been characterized by the advent of new instrumentation and knowledge (Liu 2013; Kharif et al. 2009). Instrumentation, like radars (Rosenthal and Lehner 2008; Lehner and Gunther 2004), laser scanners (Hwang et al. 2000a; Romero and Melville 2010), and stereo cameras (Wanek and Wu 2006; Benetazzo 2006; Kosnik and Dulov 2011; Benetazzo et al. 2012; Gallego et al. 2011), are indeed providing new samples of sea surface elevations $\eta$, for which the spatial dimensions $x$ and $y$ are added to the wave time records 

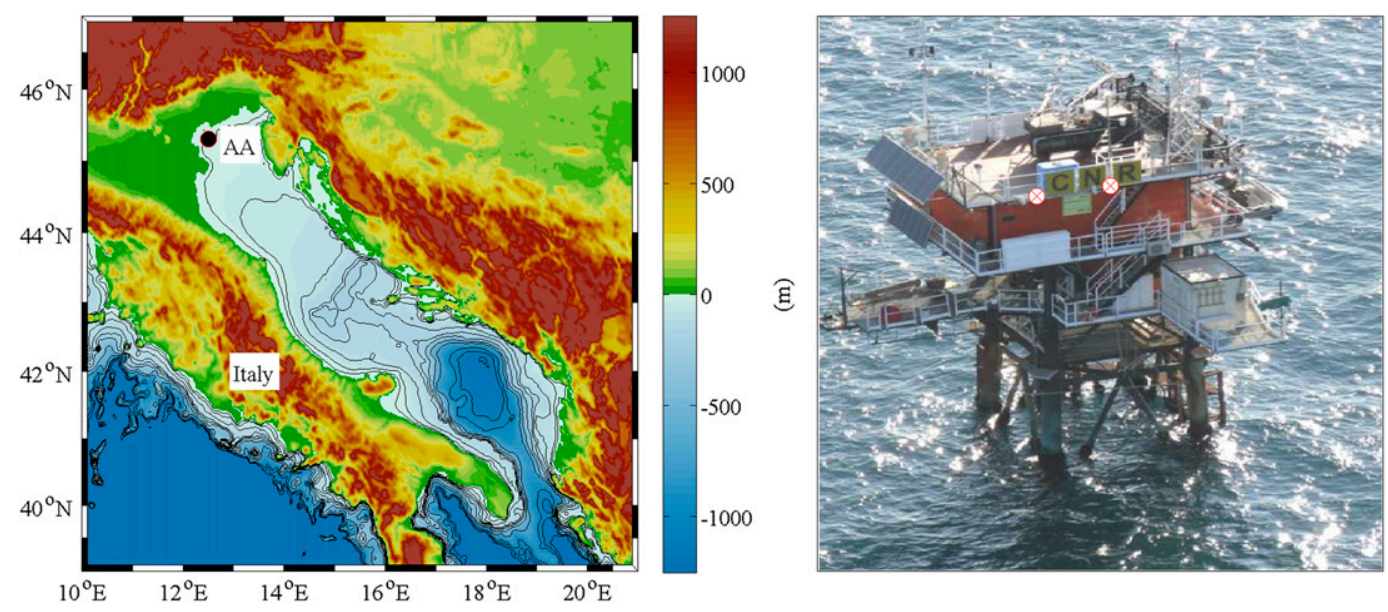

FIG. 1. (left) The Adriatic Sea bathymetry and the surrounding orography. The black-red dot shows the position of the Acqua Alta oceanographic platform (AA). (right) The platform structure and the position (white-red markers) of the two stereo cameras, looking northeastward.

$\eta(t)$, producing spatial $\eta(x, y)$ or even space-time ensembles $\eta(x, y, t)$ of wave data. These newly explored sets of observations led to new insights on sea waves behavior, including the speed of large wave crests (Banner et al. 2014), the frequency and wavenumber distributions of sea waves (Hwang et al. 2000b; Leckler et al. 2015; Romero and Melville 2010), the spatial statistics of waves (Romero and Melville 2011), and some properties of the spatiotemporal wave groups (Nieto Borge et al. 2013; Nieto Borge 2004).

A relevant application of space-time wave data is evident in the field of wave extreme analysis. Indeed, the description of large waves requires measurements capable of capturing as completely as possible the dynamics of the 3D groups (Onorato et al. 2013). Moreover, recent studies (Forristall 2006; Dysthe et al. 2008; Fedele et al. 2013) proved that wave crest maxima occurring over a portion of the sea surface are generally larger (especially for shortcrested sea conditions) than the values expected at a single point of observation. This result has also been endorsed by the conclusions of the EU-funded project "MaxWave" (Rosenthal and Lehner 2008), which suggest that criteria for modeling extreme waves must be modified and include the contribution of the spatial domain.

In the time domain, large waves are statistically described using non-Gaussian models. When the secondorder nonlinearities are dominant, the waves exhibit sharper and higher crests that are distributed according to Tayfun's model (Tayfun 1980). Second-order nonlinearities are governed by a wave steepness parameter (Fedele and Tayfun 2009) linked to the skewness coefficient of the wave record. Deviations from Tayfun's model appear when the free waves exchange energy via third-order nonlinearities; the probability and the expected value of large waves are enhanced (Tayfun and Fedele 2007; Janssen 2003) and related to the fourthorder cumulant of the surface elevation (Mori and Janssen 2006; Fedele 2008), which also contributes to the resonant wave interactions that characterize the Benjamin-Feir instability (Benjamin and Feir 1967; Onorato et al. 2001). The impact of the spatial dimension on wave maxima have been first modeled assuming that the sea states are well approximated by Gaussian multidimensional (2D space and time) random fields using Adler and Taylor's Euler characteristics approach (Adler 1981; Adler and Taylor 2007; Fedele et al. 2012) and Piterbarg's theorem (Piterbarg 1996; Krogstad et al. 2004), the latter being extended to the second order by Socquet-Juglard et al. (2005). Space-time theories model the probability of large sea elevations also accounting for the number of waves that occur within the spatial domain. In this respect, space-time models predict high probabilities for large waves, and they likely describe the extent of some extreme waves (Dysthe et al. 2008).

In our analysis, we take advantage of stereo images recorded from an oceanographic platform in the northern Adriatic Sea (Italy; see Fig. 1). Images have been processed to provide a time sequence of $2 \mathrm{D}$ snapshots of the sea surface elevation, which is thus a function of time $t$ and of the horizontal coordinates $x$ and $y$, namely, $\eta=\eta(x, y, t)$. We have analyzed the spacetime ensemble to derive the distribution of wave maxima occurring in different wave time records $\eta\left(x_{i}, y_{i}, t\right)$, selected where, on the horizontal $x-y$ plane, the 3D wave groups passing within the cameras' fields of view are close to the apex of their development. In these conditions, most probably, the wave groups produce the highest wave elevations with respect to the conditions 
of the sea state (Boccotti 2000). In particular, we have chosen the crests whose height has exceeded 1.25 times the significant wave height $\left(H_{s}\right)$, one of the common criteria used to define rogue waves (Dysthe et al. 2008).

The main focus of the presented work is to verify, by means of observational data, how large the wave crest heights may be when they are assumed to be space-time maxima and how the theoretical models used to describe extreme waves fit the empirical distribution. Outcomes are presented in the form of exceedance distribution functions of crest heights in a given record and in form of extreme values distributions. Also, the Euler characteristics approach as formulated by Fedele (2012) has been here extended to the second order for wave elevations.

The outline of the paper is as follows: Section 2 describes the stereo cameras' installation and processing and the environmental conditions during the experiment. The paper continues in section 3 with a description of the largest waves of the space-time ensemble. The empirical distribution of extreme wave crests is discussed in section 4 , where observational data are compared against maxima derived from different statistical models. Conclusions are presented in section 5 .

\section{The observational space-time ensemble}

For the purpose of the study, collection of sea surface elevation $\eta$ data (usually gathered by systems recording $\eta$ as function of time $t$ only) was extended to the sample space $(x, y)$ to produce a space-time ensemble $\eta(x, y, t)$ of the wavy sea surface. The sequence of $3 \mathrm{D}$ wave elevations was collected during an experiment conducted starting at 0940 UTC 10 March 2014 with a stereo wave imaging system [namely, Wave Acquisition Stereo System (WASS); Benetazzo 2006].

\section{a. Metocean conditions}

The WASS was mounted on top of the Acqua Alta oceanographic platform (Cavaleri 1999), located in the northernmost part of the Adriatic Sea, Italy (Fig. 1), where the sea bottom is gently sloping with a local depth $d \approx 17 \mathrm{~m}$. Stereo images were grabbed during a wellestablished east-northeast wind condition (namely, Bora
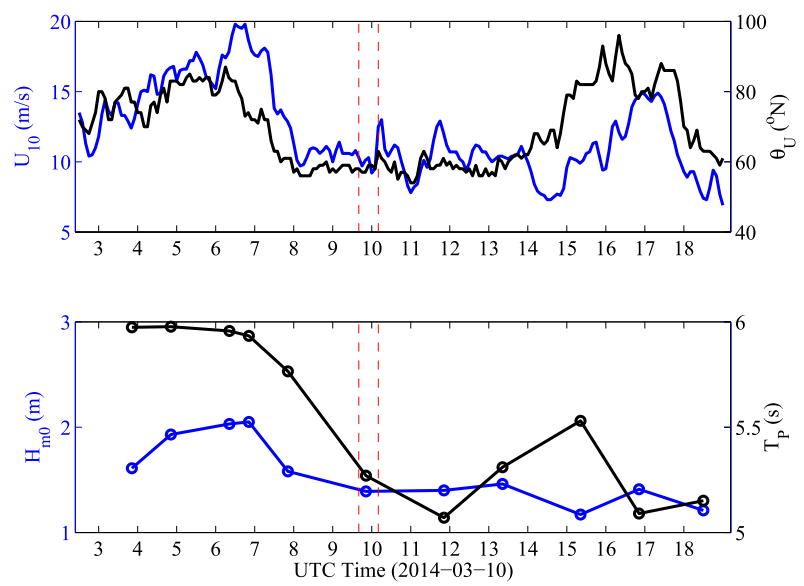

FIG. 2. Time series of wind and wave parameters from reference instrumentation at Acqua Alta on 10 Mar 2014. (top) Average wind speed $\left(U_{10}\right)$ and direction $\left(\theta_{U}\right.$, nautical convention). (bottom) Spectral significant wave height $\left(H_{m 0}\right)$ and peak period $\left(T_{p}\right)$. In both panels, the red dashed lines limit the time frame of the WASS acquisition.

wind; Benetazzo et al. 2013; see Fig. 2; Table 1), with the average wind speed (at $10-\mathrm{m}$ height) $U_{10}$ stable at about $11 \mathrm{~m} \mathrm{~s}^{-1}$ during the $2 \mathrm{~h}$ prior to the WASS acquisition. At Acqua Alta, the sea surface elevation and wave parameters are routinely measured with an acoustic surface tracking system [Nortek Acoustic Wave and Current profiler (AWAC)] at 1-Hz sampling frequency and with an accuracy of $1 \%$ of the measured value for wave elevation and $2^{\circ}$ for wave direction. During the experiment, AWAC recorded a sea state with spectral significant wave height $H_{m 0}=1.36 \mathrm{~m}$, zero-crossing significant wave height $H_{1 / 3}=1.33 \mathrm{~m}$, spectral mean period $T_{m 02}=3.8 \mathrm{~s}$, and spectral peak period $T_{p}=5.27 \mathrm{~s}$. These conditions correspond to a mature sea with a wave age $c_{p} / U_{10}=0.76$, where $c_{p}$ is the wave phase speed corresponding to $T_{p}$. At Acqua Alta, the local shoaling coefficient for the dominant waves (i.e., those with period $T_{p}$ ) was 0.97 , and the dimensionless water depth $k_{p} d$ was about 2.5 ( $k_{p}$ being the dominant wavenumber), such that we can assume as negligible the shallow-water effects on wave propagation (Holthuijsen 2008). During the WASS acquisition, the oceanic currents, measured by AWAC, were weak, experiencing a depth-averaged speed of $0.16 \mathrm{~m} \mathrm{~s}^{-1}$ (and

TABLE 1. Metocean conditions at the Acqua Alta (AA) platform on 10 Mar 2014 from 0940 to 1010 UTC. Observations from reference instrumentation (AA) and WASS data. $U_{10}$ : average wind speed; $\theta_{U}$ : average wind direction (nautical convention); $\theta_{m}:$ spectral mean direction of wave propagation; $\theta_{p}$ : spectral peak direction of wave propagation; $T_{p}$ : spectral peak wave period; $T_{m 02}$ : spectral mean wave period; $T_{z}$ : zero-crossing wave period; $H_{1 / 3}$ : zero-crossing significant wave height; and $H_{m 0}$ : spectral significant wave height.

\begin{tabular}{|c|c|c|c|c|c|c|c|c|c|}
\hline Variable & $U_{10}$ & $\theta_{U}$ & $\theta_{m}$ & $\theta_{p}$ & $T_{p}$ & $T_{z}$ & $T_{m 02}$ & $H_{1 / 3}$ & $H_{m 0}$ \\
\hline AA & $10.7 \mathrm{~m} \mathrm{~s}^{-1}$ & $59^{\circ} \mathrm{N}$ & $244^{\circ} \mathrm{N}$ & $248^{\circ} \mathrm{N}$ & $5.3 \mathrm{~s}$ & $3.8 \mathrm{~s}$ & $3.8 \mathrm{~s}$ & $1.33 \mathrm{~m}$ & $1.36 \mathrm{~m}$ \\
\hline WASS & - & - & $248^{\circ} \mathrm{N}$ & $249^{\circ} \mathrm{N}$ & $5.4 \mathrm{~s}$ & $3.8 \mathrm{~s}$ & $3.6 \mathrm{~s}$ & $1.32 \mathrm{~m}$ & $1.33 \mathrm{~m}$ \\
\hline
\end{tabular}



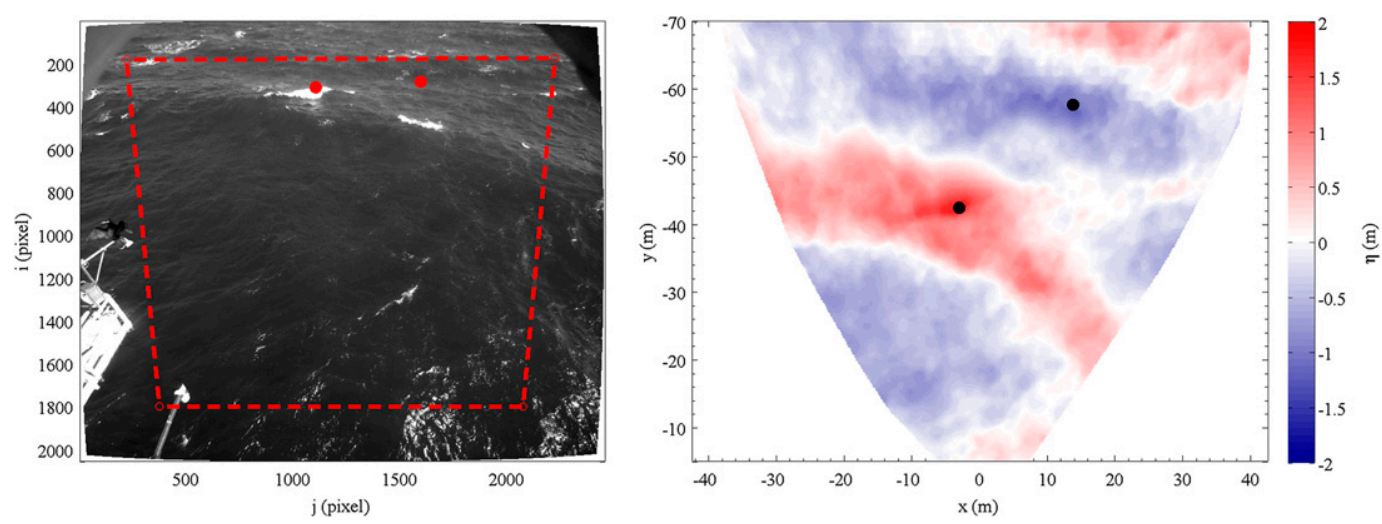

FIG. 3. (right) Example of 3D reconstruction of the sea surface elevation and (left) corresponding right camera image with superimposed locations (red dot markers) of the maximum and minimum wave elevations (black dot markers in the right panel).

equal to $0.31 \mathrm{~m} \mathrm{~s}^{-1}$ at $1 \mathrm{~m}$ underneath the mean sea surface and $0.19 \mathrm{~ms}^{-1}$ at $2 \mathrm{~m}$ ) and mostly oriented with the wind and wave direction from the sea surface through the bottom.

\section{b. The Wave Acquisition Stereo System}

The WASS setup at Acqua Alta is similar to that used in previous applications (Benetazzo et al. 2012; Leckler et al. 2015) and consisted of a pair of 5 megapixel digital cameras (with 2456 columns by 2048 rows array of $3.45-\mu \mathrm{m}$ square active elements) and mounting 5 -mm distortionless lenses, placed $2.5 \mathrm{~m}$ apart and $12.5 \mathrm{~m}$ above the mean sea level.

The cameras' internal parameters were estimated as in Albarelli et al. (2010), whereas the spatial configuration of cameras (i.e., the extrinsic parameters) was computed using an autocalibration approach based on the photometric consistency between stereo images (as in Benetazzo et al. 2014). With the device calibrated, each image pair acquired was stereo rectified and processed by a modified version of the dense stereo algorithm proposed by Hirschmüller (2008), available in the OpenCV library (http://opencv.org) by Bradski and Kaehler (2008). The semiglobal nature of the approach has the great advantage that it can relate the photometric consistency of several matching pixels to improve the reliability of the disparity map, especially for areas with loosely distinctive features. As a consequence, we can keep a relatively small window size $(13 \times 13$ pixels $)$, while still obtain a precise localization of the matches. This accounts for the matching bias for points around white-capped areas described by Leckler et al. (2015) without introducing brightness equalizations or complicated pyramidal search approaches. A discussion of the errors associated with the WASS measurements is reported in the study of Benetazzo et al. (2012), from which we recall the mean absolute quantization error of the adopted WASS setup, which is on the order of $2-3 \mathrm{~cm}$ along the three camera axes.

For each image pair, output of the stereo process is a cloud of 3D points of the sea surface elevation. For each point cloud, after being transformed to a common earth reference frame (Benetazzo 2006), a patchwise planar surface was constructed by means of 2D Delaunay triangulation. Then, the surface was resampled over a regular grid at a resolution of $0.2 \mathrm{~m}$ to span the space $x \in$ $[-42.5 \mathrm{~m}, 42.5 \mathrm{~m}]$ and $y \in[-70.0 \mathrm{~m},-5.0 \mathrm{~m}]$ (Fig. 3). The trapezoidal area of the sea surface spanned by the stereo data is about $A=2893 \mathrm{~m}^{2}$; the undisturbed surface of the sea coincides with the $x-y$ plane, the $y$ axis is turned $46^{\circ}$ clockwise from the geographical north, and the $z$ axis is pointed upward. The WASS sequence comprises 26971 image pairs, grabbed at $15 \mathrm{~Hz}$ for a total of $1798 \mathrm{~s}$ (about $30 \mathrm{~min}$ ) of data; such duration ensures reliability for waves' characterization and stationarity of the sea state (Boccotti 2000). The resulting space-time grid consists of 72325 (2D space) $\times 26971$ (time) sea surface elevation points. To limit the influence of high-frequency noise, time records $\eta\left(x_{0}, y_{0}, t\right)$ taken at each position $\left(x_{0}, y_{0}\right)$ of the $3 \mathrm{D}$ wave space-time ensemble were smoothed using a weighted linear least squares local regression and low-pass filtered at $2.0 \mathrm{~Hz}$ (Fig. 4).

\section{c. Space-time wave data}

Assuming the observed sea condition was stationary in time and homogenous in space, the frequency-direction wave spectrum $S(f, \theta)$ was computed using a stochastic approach, namely, the extended maximum entropy method (EMEP; Hashimoto et al. 1994). The frequencydirection spectrum was resolved with 180 equally spaced directions to cover the full circle and 1024 equally distributed frequencies from 0.05 to $2 \mathrm{~Hz}$. The EMEP method has already been successfully applied to WASS 

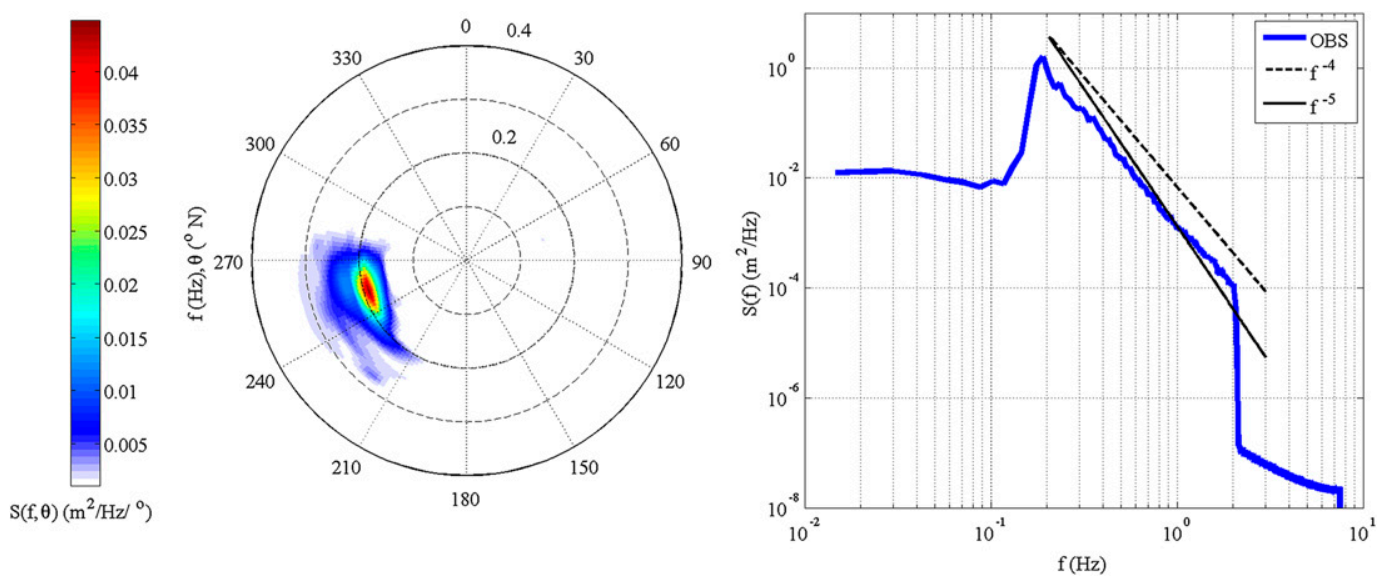

FIG. 4. (left) Frequency-direction spectrum and (right) omnidirectional frequency spectrum estimated from stereo wave data (OBS). In the right panel, the dashed and solid black lines are reference spectral slopes.

data (Fedele et al. 2013; Barbariol et al. 2014) and preferred here to the 3D Fourier transform of the spacetime ensemble $\eta(x, y, t)$ that otherwise would have provided a small directional resolution around the spectral peak (Hwang et al. 2000b). The directional spectrum $S(f, \theta)$ shown in Fig. 4 is unimodal with $H_{m 0}=$ $1.33 \mathrm{~m}$, peak direction of wave propagation $\theta_{p}=249^{\circ} \mathrm{N}$, and peak frequency $f_{p}=0.19 \mathrm{~Hz}$. The directional spreading of $S(f, \theta)$ integrated over the frequencies, estimated according to Kuik et al. (1988), is equal to $39^{\circ}$, a characteristic value of wind sea states.

An estimate of the mean $\langle\eta\rangle=\langle\eta(x, y, t)\rangle$ of the dataset is $-0.001 \mathrm{~m}$, where the angle brackets denote space-time averaging. The standard deviation $\sigma$ and the coefficients of skewness $\lambda_{3}$ and kurtosis $\lambda_{4}$ have been estimated from the second-, third-, and fourth-order moments of the surface displacement $\eta(x, y, t)$ probability density function (PDF) as follows:

$$
\begin{aligned}
\sigma & =\left\langle[\eta(x, y, t)-\langle\eta\rangle]^{2}\right\rangle^{1 / 2}, \\
\lambda_{3} & =\frac{\left\langle[\eta(x, y, t)-\langle\eta\rangle]^{3}\right\rangle}{\sigma^{3}}, \text { and } \\
\lambda_{4} & =\frac{\left\langle[\eta(x, y, t)-\langle\eta\rangle]^{4}\right\rangle}{\sigma^{4}} .
\end{aligned}
$$

The observed values for these parameters are $\sigma=0.334 \mathrm{~m}$, $\lambda_{3}=0.16$, and $\lambda_{4}=3.22$. The significant wave height $H_{\mathrm{s}}$, taken as 4 times the standard deviation $\sigma$ of the surface elevation, is $1.34 \mathrm{~m}$.

In a previous study (Benetazzo et al. 2012), WASS data have already been validated through a comparison with observations from other instruments. Here, for the sake of assessment, sea state parameters estimated using the space-time ensemble have been compared against observations from the AWAC instrument. Results in
Table 1 show that WASS observations are consistent with the measurements collected from AWAC, with small differences on the order of a few centimeters for wave heights and a few tenths of a second for wave periods.

\section{An observatory for large waves}

\section{a. Recognition of time records with the highest waves}

With the purpose of analyzing the highest waves of the space-time ensemble ENS, it is remarkable to note that the maximal sea surface elevation $\eta_{\mathrm{ENS}, \max }=\max [\eta(x, y, t)]$ is $2.12 \mathrm{~m}$, about 6.4 times larger than the standard deviation $\sigma$, and it exceeds $H_{s}$ by a factor of 1.59. We have therefore examined the entire ensemble to isolate the $3 \mathrm{D}$ wave groups, passing through the stereo camera field of view, whose maximum elevation $\eta_{c}$ has exceeded a specific value, which we have chosen corresponding to the rogue wave threshold for crest heights, namely, 1.25H $H_{s}$ (Haver 2004; Dysthe et al. 2008). Our selected threshold is somehow arbitrary and adopted with the only purpose of isolating the highest waves within the ensemble. Additionally, we have visually checked that the selected wave maxima were not contained in areas of large breaking foam, where the images tend to saturate, thus lowering the photometric consistency of the stereo pair. Also, data analysis has been limited to the central part of the 2D elevation maps to prevent reconstruction biases due to the image borders.

In this way, we have identified 23 independent 3D wave crests (e.g., the crest shown in Fig. 3 at $x \approx-3 \mathrm{~m}$ and $y \approx-42 \mathrm{~m})$ at instants $t_{i}$ and spatial locations $\left(x_{i}, y_{i}\right)$ shown in Fig. 5, most likely when and where the crests traveled close to the center of the 3D wave groups (Boccotti 2000). We note that the wave crest positions 


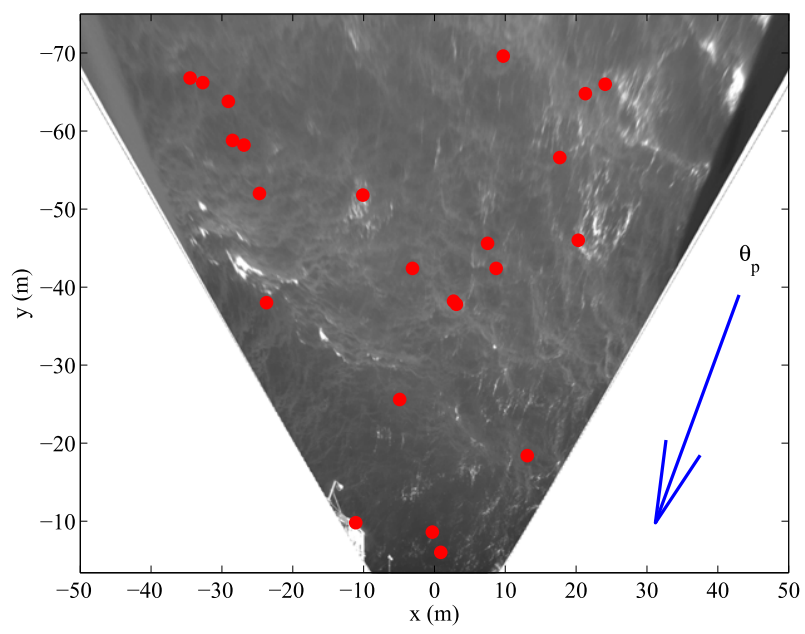

FIG. 5. Orthorectified right camera image plane within the stereo-matched area and locations (red dots) of the 23 wave records $\eta_{i}(t)=\eta\left(x_{i}, y_{i}, t\right)$, such that $\max \left[\eta_{i}(t)\right]>1.25 H_{s}$. The blue arrow shows the peak direction of wave propagation $\left(\theta_{p}\right)$ with respect to the camera axes.

displayed in Fig. 5 are distributed all over the spatial coverage, fulfilling the assumption of homogeneity of the wave process. The fixed $\left(x_{i}, y_{i}\right)$ positions of these maximal crests have therefore been assumed to act as virtual pointlike probes of the space-time ensemble (see Fig. 9 of Benetazzo et al. 2012). At these points, we have extracted the corresponding time series $\eta_{i}(t)=\eta\left(x_{i}, y_{i}, t\right)$, with $i=1,2, \ldots, 23$. As the sea state under investigation was generated by a Bora wind condition, we have labeled the time records as $B_{i}$ (Table 2) and ordered them in descending order according to the maximum crest height of the record $\eta_{i, \max }=\max \left[\eta_{i}(t)\right]$.

Several considerations can be drawn from the wave data of records $B_{i}$. First, records have been analyzed via zero-crossing analysis to isolate single crest heights and wave heights as crest-to-trough vertical distance. The average $\left(H_{1 / 3}\right)$ of the highest one-third of the waves from all records $B_{i}$ is $1.32 \mathrm{~m}$; therefore, the average is comparable (albeit smaller) to $H_{s}$. In the following analysis, however, we will retain $H_{s}=4 \sigma$ instead of $H_{1 / 3}$, as a conservative upper bound to estimate dimensionless crest and wave heights. We have therefore examined each record in proximity to the maximal crest height $\eta_{i \text { max }}$. In this respect, the time portrait of the highest wave of the ensemble (belonging to the record $B_{1}$ and having $\left.\eta_{c}=\eta_{\mathrm{ENS} \text {,max }}\right)$ is displayed in Fig. 6 , where the nature of this large wave is visible within the wave group passing through the position $\left(x_{1}, y_{1}\right)$ from 610 to $640 \mathrm{~s}$. In the record $B_{1}$, increasing high waves do not precede the highest wave that seems to appear out of nowhere, whereas the spatial continuity is fulfilled (see for example the crest in Fig. 3). The time profile of the wave is
TABLE 2. Parameters of the individual maximal waves of the records $B_{i}$ and average values (Avg). Wave index $i$ (from 1 to 23) corresponds to the record number ordered with decreasing $\eta_{i, \max }=$ $\max \left[\eta_{i}(t)\right] . H_{i, \max }$ : maximum wave height; $\varepsilon_{i, \max }$ : steepness of the maximal wave; and $H_{s}$ : significant wave height.

\begin{tabular}{cccc}
\hline \hline Record & $\eta_{i, \max } / H_{s}$ & $H_{i, \max } / H_{s}$ & $\varepsilon_{i, \max }$ \\
\hline$B_{1}$ & 1.59 & 2.22 & 0.36 \\
$B_{2}$ & 1.52 & 2.39 & 0.36 \\
$B_{3}$ & 1.49 & 2.24 & 0.26 \\
$B_{4}$ & 1.48 & 2.21 & 0.40 \\
$B_{5}$ & 1.47 & 2.19 & 0.36 \\
$B_{6}$ & 1.45 & 2.10 & 0.36 \\
$B_{7}$ & 1.45 & 2.42 & 0.35 \\
$B_{8}$ & 1.43 & 1.93 & 0.30 \\
$B_{9}$ & 1.41 & 2.16 & 0.36 \\
$B_{10}$ & 1.38 & 1.86 & 0.36 \\
$B_{11}$ & 1.38 & 2.26 & 0.29 \\
$B_{12}$ & 1.37 & 1.79 & 0.28 \\
$B_{13}$ & 1.36 & 1.99 & 0.29 \\
$B_{14}$ & 1.34 & 2.11 & 0.41 \\
$B_{15}$ & 1.34 & 2.03 & 0.38 \\
$B_{16}$ & 1.32 & 2.05 & 0.34 \\
$B_{17}$ & 1.32 & 2.07 & 0.33 \\
$B_{18}$ & 1.31 & 1.92 & 0.33 \\
$B_{19}$ & 1.31 & 1.93 & 0.31 \\
$B_{20}$ & 1.28 & 1.98 & 0.34 \\
$B_{21}$ & 1.27 & 2.01 & 0.34 \\
$B_{22}$ & 1.26 & 2.06 & 0.45 \\
$B_{23}$ & 1.25 & 1.96 & 0.31 \\
Avg & 1.38 & 2.08 & 0.34 \\
\hline & & &
\end{tabular}

asymmetric and skewed around the peak with a maximum crest-to-trough height $H_{1, \max }=2.96 \mathrm{~m}$, about 2.22 times larger than $H_{s}$. The plot of the individual highest waves of all records $B_{i}$ around $\eta_{i \text { max }}$ is shown in Fig. 6 . These large waves have sharper and narrower crests than the other waves of the group and tend to assume a deterministic profile (Boccotti 2000; Tayfun and Fedele 2007), which was proved to be in very good agreement with experimental data (Fedele et al. 2013). The average period of the largest waves of the records is $4.7 \mathrm{~s}, 13 \%$ smaller than the peak period. The waves adjacent (next or previous) to the maximal ones have a broader time profile with crest heights generally one-third $\left(\sim 0.4 H_{s}\right.$ compared to $\sim 1.4 H_{s}$ ) of the highest wave of the group, as also displayed by the well-known Draupner and Andrea rogue waves (Magnusson and Donelan 2013). As discussed hereinafter, even if the smaller crests of the group have heights that are well fitted by the theoretical distributions for wave crest heights, the highest one is a real outlier from the standard statistics.

Some parameters describing the largest waves of the records $B_{i}$ are reported in Table 2. Within the data presented, it is worth noting that 6 out of 23 records have a maximal wave height $H_{i, \max }$ exceeding $H_{s}$ by a factor of 2.2 and 16 exceed $H_{s}$ by a factor of 2.0. The 

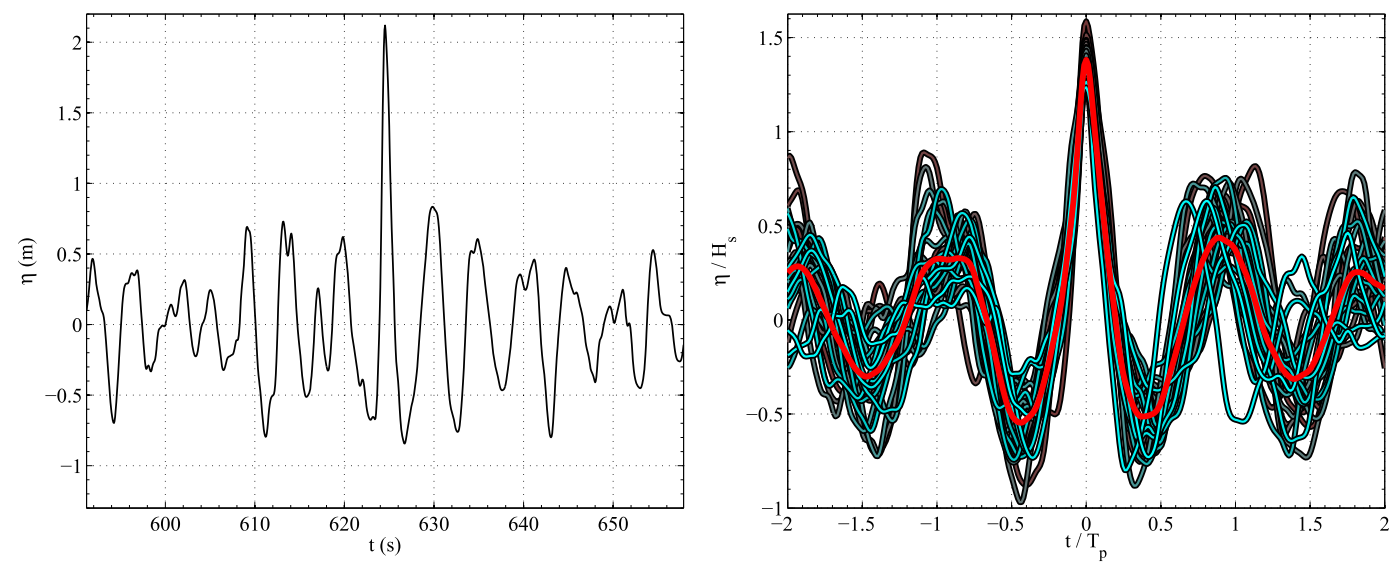

FIG. 6. (left) Extract of the dimensional sea surface elevation $\eta_{1}(t)=\eta\left(x_{1}, y_{1}, t\right)$ of the record $B_{1}$ around $\max \left[\eta_{1}(t)\right]$ and (right) dimensionless sea surface elevation for all records $B_{i}$ around $\max \left[\eta_{i}(t)\right]$. In the right panel, the mean wave profile is shown as red line, and, for graphical purposes, the time axis is centered at the moment when $\max \left[\eta_{i}(t)\right]$ occurs and extended to include $4 T_{p}$.

average maximal crest and wave heights are $1.38 H_{s}$ and $2.08 H_{s}$, respectively. For reference, the abnormal Draupner wave (Magnusson and Donelan 2013; Haver 2004) had $\eta_{\max }=1.55 H_{s}$ and $H_{\max }=2.15 H_{s}$. The local steepness of the highest wave of the records, defined as $\varepsilon_{i, \max }=$ $\eta_{i, \max } k_{i, \max }$ (where $k_{i, \max }$ is the wavenumber corresponding to the period of the wave with $\eta_{c}=\eta_{i \text {,max }}$ ), is given in Table 2. The steepness is 0.34 on average and 0.45 at most for record $B_{22}$, where, from visual inspection of the stereo images, the maximal crest was at the breaking onset. We also note that not all the highest waves of the records were in incipient breaking conditions.

The PDF of the zero-mean and dimensionless wave surface elevations $\eta_{i}(t)$ for the $23 B_{i}$ records aggregated (for a total of about 620000 sea elevation data) is provided in Fig. $7\left(\mathrm{OBS}_{B}\right)$, with the Gaussian distribution and the nonlinear third- and fourth-order corrections (Longuet-Higgins 1963), respectively given by

$$
\begin{aligned}
p_{G}(z=\eta / \sigma)= & \frac{1}{\sqrt{2 \pi}} \exp \left(-\frac{z^{2}}{2}\right) \\
p_{G-C 3}(z=\eta / \sigma)= & \frac{1}{\sqrt{2 \pi}} \exp \left(-\frac{z^{2}}{2}\right)\left[1+\frac{\lambda_{3}}{6}\left(z^{3}-3 z\right)\right] \\
p_{G-C 4}(z=\eta / \sigma)= & \frac{1}{\sqrt{2 \pi}} \exp \left(-\frac{z^{2}}{2}\right)\left[1+\frac{\lambda_{3}}{6}\left(z^{3}-3 z\right)\right. \\
& \left.+\frac{\lambda_{4}-3}{24}\left(z^{4}-6 z^{2}+3\right)\right]
\end{aligned}
$$

plotted for reference. For the nonlinear corrections, the skewness [(2)] and kurtosis [(3)] estimated from the ensemble of the space-time data have been adopted. The deviation of the positive tail of the empirical PDF from the theoretical distributions (that otherwise fit well the observations) starts at elevations of about $4 \sigma\left(=H_{s}\right)$. Even the corrections of the Gaussian model cannot reproduce the extreme wave crests observed in the large elevation region. On the contrary, once the probabilities are computed using the whole space-time ensemble ( $\mathrm{OBS}_{\mathrm{ENS}}$ in Fig. 7), the $G-C 4$ approximation fits the empirical PDF well.

To summarize, the ensemble of the wave records $B_{i}$ comprises a sample of time-varying sea surface displacements observed at fixed spatial locations, chosen where the 3D wave groups have exhibited a maximal elevation. Hence, the $\left(x_{i}, y_{i}\right)$ position of each record is a preferential site for wave observation, as the surface area retrieved by the cameras allows the observer to follow the wave groups in their spatial evolution and to freeze the sea surface when the wave groups are close to the apex of their development and display large amplitude waves. So constrained, wave records $B_{i}$ hold the largest crest heights among all those observable within the space-time ensemble.

\section{b. Distribution of crest and wave heights of the record $B_{1}$}

As stated in section 3a, the maximal crest height of each record $B_{i}$ corresponds to a very large displacement compared to the variance of the sea state. In this respect, a zero-crossing analysis of single waves of the record $B_{1}$ reveals some insights into the probability of occurrence of the largest wave. The exceedance distribution functions (EDF; hereinafter represented as $P\{\}$ ) of the crest $\eta_{c}$ and wave heights $H$ of record $B_{1}$ are presented in Fig. 8, where the estimates and the stability band of EDF have been derived from mean and standard deviation [Eq. (16) of Tayfun and Fedele 2007] of the $j$ th value of the order statistics. It is worth noting that two single waves of the 


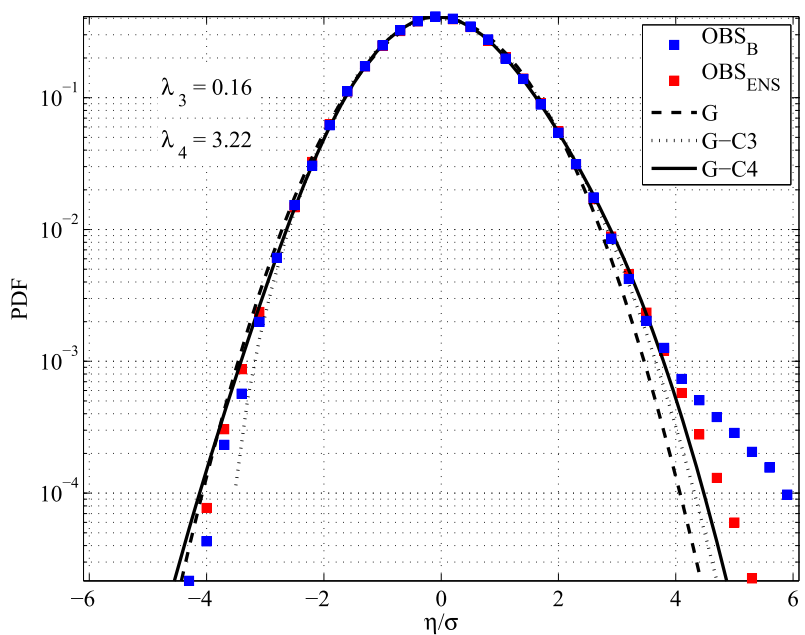

FIG. 7. PDF of dimensionless sea surface elevations of the records $B_{i}\left(\mathrm{OBS}_{B}\right)$ and of the space-time ensemble $\left(\mathrm{OBS}_{\mathrm{ENS}}\right)$. The Gaussian distribution $(G)$, the third- $(G-C 3)$, and fourth-order $(G$ $C 4)$ nonlinear corrections are displayed for reference.

record display large $\eta_{c}$ and $H$, with exceedance levels that deviate strongly from those of the remaining waves. A question arises whether existing theoretical models predict these probability levels.

First, for a sea state such that the surface elevation is Gaussian distributed and surface waves are narrowbanded in frequency, the wave crest heights gathered at a fixed point have an EDF given by the Rayleigh formula (hereinafter R; Longuet-Higgins 1952)

$$
P_{\mathrm{R}}\left\{\eta_{\mathrm{c}} / \sigma>z\right\}=\exp \left(-z^{2} / 2\right)
$$

that underpredicts the empirical probabilities of crest heights (Fig. 8) because of nonlinear effects occurring in realistic active sea states.

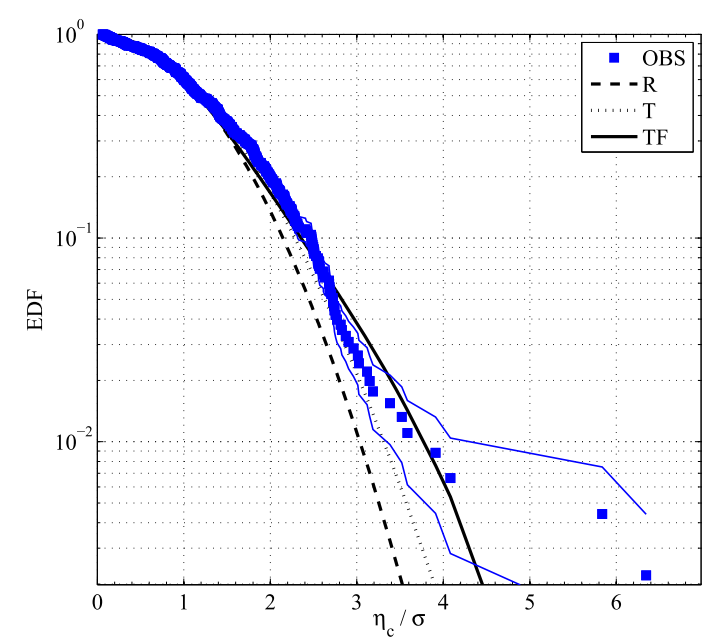

The second-order correction in the Stokes expansion of the linear model affects the probability distribution of $\eta_{c}$, which, here, is expressed in the Tayfun form (hereinafter T; Tayfun 1980), namely,

$$
P_{\mathrm{T}}\left\{\eta_{c} / \sigma>\xi\right\}=\exp \left(-z^{2} / 2\right),
$$

where $\xi$ is the second-order elevation. In the T model, the variable $z$ satisfies the quadratic equation

$$
\xi=z+\frac{\mu}{2} z^{2} \rightarrow z=\frac{-1+\sqrt{1+2 \mu \xi}}{\mu},
$$

where $\mu$ is the wave steepness. Following Fedele and Tayfun (2009), the wave steepness has been evaluated as a statistically stable estimate from the zeroth- $\left(m_{000}\right)$, first- $\left(m_{001}\right)$, and second-order $\left(m_{002}\right)$ moments of the directional spectrum given by

$$
m_{i j l}=\iint k_{x}^{i} k_{y}^{j} f^{l} S(f, \theta) d f d \theta,
$$

where $\mathbf{k}=\left(k_{x}, k_{y}\right)$ is the wavenumber vector associated with the frequency $f$ (through the linear dispersion relation for sea waves) and direction $\theta$. The steepness is expressed as

$$
\mu=\mu_{m}\left(1-\nu+\nu^{2}\right),
$$

where $\mu_{m}=\sigma\left(2 \pi m_{001} / m_{000}\right)^{2} / g$ is an integral measure of the wave steepness (Fedele and Tayfun 2009), corrected with the spectral bandwidth $\nu=\sqrt{m_{000} m_{002} / m_{001}^{2}-1}$ (Longuet-Higgins 1975). The observed values for these parameters are $\mu=0.06$ and $\nu=0.50$. In Fig. 8 , the $\mathrm{T}$ model tends to underestimate the experimental data for

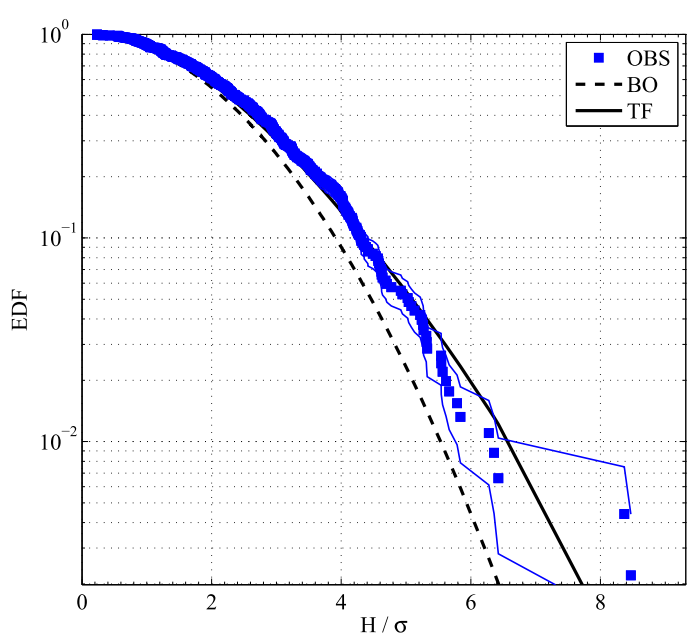

FIG. 8. Probability of exceedance (EDF) of (left) dimensionless crest heights and (right) wave heights of the record $B_{1}$ (OBS). The empirical EDF stability band is plotted as blue solid line. Reference distributions: Rayleigh (R), Tayfun (T), Tayfun-Fedele (TF), and Boccotti (BO). 
crest heights larger than $2 \sigma$, similar to what was found by Fedele (2008) for steepness of about 0.07.

At third-order correction, interactions between free waves produce a deviation from Gaussian and secondorder theories (e.g., Janssen 2003). In this respect, the Tayfun-Fedele approximation (hereinafter TF; Tayfun and Fedele 2007) gives the EDF of dimensionless wave crest heights as

$$
\begin{aligned}
& P_{\mathrm{TF}}\left\{\eta_{c} / \sigma>\xi\right\} \\
& \quad=\exp \left[-\frac{1}{2}\left(\frac{-1+\sqrt{1+2 \mu \xi}}{\mu}\right)^{2}\right]\left[1+\frac{\Lambda}{64} \xi^{2}\left(\xi^{2}-4\right)\right],
\end{aligned}
$$

where the variable $\Lambda$ is a function of the fourth-order joint cumulants of the sea surface elevation and its conjugate [Eq. (42) of Tayfun and Fedele 2007], and for the record $B_{1}$ it takes the value 1.09 . The TF model increases the probability of large waves over that given by the $\mathrm{R}$ and $\mathrm{T}$ models and well reproduces the empirical distribution of wave crests in Fig. 8, except the two extreme waves that occur on the tail of the empirical EDF.

As far as the observed wave height EDF is concerned (right panel of Fig. 8), for linear waves the Rayleigh model overestimates the probability levels as it does not account for finite bandwidth effects (Tayfun 1981; Boccotti 2000; Forristall 1978), which are included in the asymptotic linear distribution derived by Boccotti (2000; hereinafter BO):

$$
P_{\mathrm{BO}}\{H / \sigma>h\}=\exp \left[-\frac{1}{4\left(1+\Psi^{*}\right)} h^{2}\right],
$$

where $\Psi^{*}$ is the absolute value of the ratio between the global minimum and the global maximum of the autocovariance function of the record. For the record $B_{1}$ the parameter $\Psi^{*}=0.66$ (belonging to the typical range for wind waves; Boccotti 2000). Second-order nonlinearities have negligible effects to the crest-to-through wave heights (Tayfun and Fedele 2007). At a higher order of approximation, the TF model for wave height is expressed as

$$
P_{\mathrm{TF}}\{H / \sigma>h\}=\exp \left(-\frac{1}{8} h^{2}\right)\left[1+\frac{\Lambda}{1024} h^{2}\left(h^{2}-16\right)\right],
$$

which improves the prediction of the $\mathrm{BO}$ model in Fig. 8 . For narrowbanded sea states, $\Lambda \approx 8 / 3\left(\lambda_{4}-3\right)$, showing a direct dependence of the probability levels on the kurtosis coefficient (Tayfun and Fedele 2007; Mori and Janssen 2006). In this respect, the kurtosis [(3)] of the space-time data $\left(\lambda_{4}=3.22\right)$ is different from the corresponding value of the wave record $B_{1}\left(\lambda_{4, B 1}=3.52\right)$.
The higher kurtosis of the record $B_{1}$ is a direct consequence of the fact that significantly large displacements occur in the proximity of the highest wave. In fact, removing this single wave from the series and recalculating the kurtosis of the remaining record, we have found a kurtosis of 3.21, very close to the ensemble value.

Crest and wave heights distributions displayed in Fig. 8 for the record $B_{1}$ are typical of each record $B_{i}$, where the highest waves are real outliers of the standard statistics. These unusual waves will be discussed in section 4 in the context of extreme values statistics, also including space-time effects.

\section{Statistics of extremes}

\section{a. Empirical distribution}

The extreme crest heights $\eta_{i, \text { max }}$ are members of an ordered sample of the largest sea surface elevations within the space-time ensemble. In this respect, some studies (Fedele 2012; Fedele et al. 2013; Forristall 2011; Krogstad et al. 2004; Dysthe et al. 2008) documented that wave maximal crests occurring over an area are generally larger than those expected (on average) at a given point inside that area, especially for short-crested sea waves. Distributions of such space-time extremes were derived from theories developed to describe multidimensional random fields, in which the wave crests are labeled as maxima exceeding a given threshold (Adler 1981; Adler and Taylor 2007; Piterbarg 1996). In fact, the concept of a single 3D "wave" is per se ambiguous, as a generalization of the zero-crossing procedure to higher dimensions is far from being trivial (Fedele et al. 2012; Worsley 1996).

In the present study, the empirical distribution of wave extremes relies on the maximal crest height $\eta_{i, \text { max }}$ of each record $B_{i}$ (Table 2$)$ : $\left(\eta_{1, \max }, \eta_{2, \max }, \ldots, \eta_{23, \text { max }}\right)$. These extremes, homogeneously distributed within the stereo camera field of view, are assumed to pertain to, on average, a sea surface area $A / 23=126 \mathrm{~m}^{2}$, equivalent to a square with sides $X=Y=11.2 \mathrm{~m}$. The duration $D$ of the sea state is $1798 \mathrm{~s}$, such that the average number of waves in time is $N=D / T_{m 02}=498$. The set of $\eta_{i, \max }$ variables defines a stochastic process, where each $\eta_{i \text {,max }}$ is assumed to be an independent realization of a large wave crest (Boccotti 2000). The empirical EDF of the maximal crests is plotted in Fig. 9; the expected maxi$\operatorname{mum}\left\langle\eta_{i, \max }\right\rangle$ is $1.85 \mathrm{~m}$ (equivalent to $5.52 \sigma$ and $1.38 H_{s}$ ) with a standard deviation of $0.12 \mathrm{~m}$.

\section{b. Theoretical distributions}

The empirical distribution of maxima is compared against theoretical EDFs of extreme crest heights, which stem from initial distributions of individual crest heights 


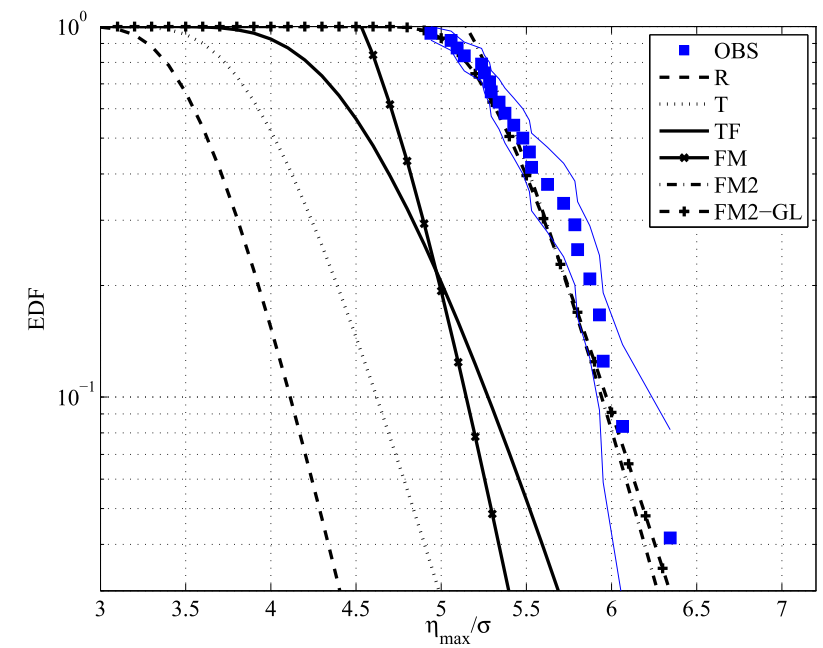

FIG. 9. Probability of exceedance (EDF) of dimensionless observed extreme crest heights $\eta_{i, \max }=\max \left[\eta_{i}(t)\right]$ of the records $B_{i}$ (OBS). The empirical EDF stability band is plotted as blue solid line. Reference distributions of extremes: Rayleigh (R); Tayfun (T); Tayfun-Fedele (TF); Fedele (FM); second-order FM (FM2); and asymptotic Gumbel limit of FM2 (FM2-GL). The reference duration is $D=1798 \mathrm{~s}$. Space-time extremes are computed over an area $X Y=11.2 \times 11.2 \mathrm{~m}^{2}=126 \mathrm{~m}^{2}$.

in a given record. In the linear and narrowband approximation, the probability that in a time record of $N$ waves the maximal crest height $\eta_{\max }$ exceeds a given threshold $z$ is given in the general form (Gumbel 1958)

$$
P_{\mathrm{R}, \max }\left\{\eta_{\max } / \sigma>z \mid N\right\}=1-\left(1-P_{\mathrm{R}}\right)^{N},
$$

where $P_{\mathrm{R}}$ follows from the Rayleigh distribution (5). The asymptotic Gumbel limit of (13) permits an estimation of the expected value of the maximum crest height in $N$ waves as (Cartwright and Longuet-Higgins 1956)

$$
E_{\mathrm{R}, \max } / \sigma \approx h_{\mathrm{R}}+\frac{\gamma}{\alpha_{\mathrm{R}}}=\left(1+\frac{\gamma}{2 \ln N}\right) \sqrt{2 \ln N},
$$

where $h_{\mathrm{R}}$ is the most probable value (viz., the mode) and $\alpha_{\mathrm{R}}$ is the intensity function, both depending on the initial distribution and the sample size. The parameter $\gamma \approx 0.5772$ is the Euler-Mascheroni constant. According to Gumbel (1958), the standard deviation of the largest values is $\pi /\left(\sqrt{6} \alpha_{\mathrm{R}}\right)$. From the Rayleigh model, the expected maximum crest height for $N=$ 498 waves is $E_{\mathrm{R}, \max } \approx 1.23 \mathrm{~m}$ (equivalent to $3.68 \sigma$ and $\left.0.92 H_{s}\right) \pm 0.12 \mathrm{~m}$, which is smaller than the observed value (Fig. 9). Accounting for second-order nonlinearities, the EDF of crest maxima derived from the Tayfun model (6) is

$$
P_{\mathrm{T}, \max }\left\{\eta_{\max } / \sigma>\xi \mid N\right\}=1-\left(1-P_{\mathrm{T}}\right)^{N} .
$$

The mean of the maximal crests is approximated as (Tayfun and Fedele 2007)

$$
E_{\mathrm{T}, \max } / \sigma \approx\left(h_{\mathrm{R}}+\frac{\mu}{2} h_{\mathrm{R}}^{2}\right)+\frac{\gamma}{h_{\mathrm{R}}}\left(1+\mu h_{\mathrm{R}}\right),
$$

which results equal to $1.37 \mathrm{~m}$ (equivalent to $4.10 \sigma$ or $\left.1.03 H_{s}\right) \pm 0.18 \mathrm{~m}$ and underestimates the observations of about $1.5 \sigma$. At the next order of approximation, the TF model [(10)] provides the initial distribution for the EDF of maximal wave crests in trains of $N$ waves

$$
P_{\mathrm{TF}, \max }\left\{\eta_{\max } / \sigma>\xi \mid N\right\}=1-\left(1-P_{\mathrm{TF}}\right)^{N},
$$

for which the expected value is written in the general form as

$$
E_{\mathrm{TF}, \mathrm{max}} / \sigma \approx h_{\mathrm{TF}}+\frac{\gamma}{\alpha_{\mathrm{TF}}} .
$$

The modal value $h_{\mathrm{TF}}$ satisfies

$\exp \left[-\frac{1}{2}\left(\frac{-1+\sqrt{1+2 \mu h}}{\mu}\right)^{2}\right]\left[1+\frac{\Lambda}{64} h^{2}\left(h^{2}-4\right)\right]=1 / N$,

and the intensity function $\alpha_{\mathrm{TF}}$ is given by

$$
\alpha_{\mathrm{TF}}=\frac{1}{\mu}\left(1-\frac{1}{\sqrt{1+2 \mu h_{\mathrm{TF}}}}\right)-\frac{\frac{\Lambda}{8}\left(\frac{h_{\mathrm{TF}}^{3}}{2}-h_{\mathrm{TF}}\right)}{1+\frac{\Lambda}{64}\left(h_{\mathrm{TF}}^{4}-4 h_{\mathrm{TF}}^{2}\right)} .
$$

The mean value of $\Lambda$ over the wave records $B_{i}$ is 0.83 . The TF model improves the prediction of the $\mathrm{R}$ and $\mathrm{T}$ models (Fig. 9), even though the average expected maximum $E_{\mathrm{TF}, \max } \approx 1.56 \mathrm{~m}$ (equivalent to $4.66 \sigma$ and $\left.1.17 H_{s}\right) \pm 0.18 \mathrm{~m}$ is still smaller than the observed value of about $\sigma$.

The theoretical R, T, and TF models assume the sea surface elevation recorded in time at a given position; within the time record, single waves are isolated using a zero-crossing procedure. As records $B_{i}$ are extracted from a space-time ensemble, we have also compared the empirical EDF against those derived from stochastic distributions of maxima over multidimensional fields. The extension of the linear $\mathrm{R}$ model to spacetime ensembles requires the generalization of the 
concept of waves in a multidimensional domain, which in this study spans two spatial dimensions (over a sea surface area of sides $X$ and $Y$ ) and a time interval $D$. We refer here to the studies of Piterbarg (1996) and Adler and Taylor (2007) who computed the probability of maxima in generic random fields. Drawing upon the results of Fedele (2012; hereinafter FM) derived from
Adler and Taylor (2007) for a stationary and homogeneous Gaussian wave field $\eta(x, y, t)$ bounded by a space-time volume $V=X Y D$ (see Fig. 1 of Fedele 2012), one has to first compute the average number of waves within $V\left(N_{V}\right)$, on the boundary surfaces $S$ of $V\left(N_{S}\right)$, and along the perimeter $B$ of $S\left(N_{B}\right)$, which are given by

$$
\begin{aligned}
& N_{V}=2 \pi \frac{X Y D}{L_{x} L_{y} T_{m}} \sqrt{1-\alpha_{x t}^{2}-\alpha_{x y}^{2}-\alpha_{y t}^{2}+2 \alpha_{x t} \alpha_{x y} \alpha_{y t}} \\
& N_{S}=\sqrt{2 \pi}\left(\frac{X D}{L_{x} T_{m}} \sqrt{1-\alpha_{x t}^{2}}+\frac{X Y}{L_{x} L_{y}} \sqrt{1-\alpha_{x y}^{2}}+\frac{Y D}{L_{y} T_{m}} \sqrt{1-\alpha_{y t}^{2}}\right) \\
& N_{B}=\frac{X}{L_{x}}+\frac{Y}{L_{y}}+\frac{D}{T_{m}}
\end{aligned}
$$

The mean zero-crossing period $\left(T_{m}=T_{m 02}\right)$, the mean zero-crossing wavelength and wave crest length $\left(L_{x}=\right.$ $L_{x m 02}$ and $L_{y}=L_{y m 02}$, respectively), and the irregularity parameters of the sea state $\left(\alpha_{x t}, \alpha_{y t}\right.$, and $\alpha_{x y}$, all ranging between -1 and +1$)$ are computed from the moments [(8)] of the directional spectrum $S(f, \theta)$ as (Baxevani and Rychlik 2006; Fedele 2012)

$T_{m}=\sqrt{\frac{m_{000}}{m_{002}}}, \quad L_{x}=2 \pi \sqrt{\frac{m_{000}}{m_{200}}}, \quad L_{y}=2 \pi \sqrt{\frac{m_{000}}{m_{020}}}, \quad$ and

$\alpha_{x t}=\frac{m_{101}}{\sqrt{m_{200} m_{002}}}, \quad \alpha_{y t}=\frac{m_{011}}{\sqrt{m_{020} m_{002}}}, \quad \alpha_{x y}=\frac{m_{110}}{\sqrt{m_{200} m_{020}}}$.

The irregularity parameters control the number of waves [(21)] and play an important role in the distribution of the highest crests. For instance, assuming $x$ as the mean direction of wave propagation, $\alpha_{x t} \approx 1$ in narrowband sea states, such that the numbers of waves $N_{S}$ and $N_{V}$ decrease to preserve stochastic independence among waves (Baxevani and Rychlik 2006).

Drawing upon Adler and Taylor (2007), the exceedance probabilities that one of the $3 \mathrm{D}$ wave crest heights (normalized by $\sigma$ ) exceeds the threshold $z$ within the volume $V$, on the boundary surfaces $S$ and along the perimeter $B$ are expressed, respectively, as

$$
\begin{aligned}
P_{B}\left\{\eta_{c} / \sigma>z \mid B\right\} & =P_{\mathrm{R}} \\
P_{S}\left\{\eta_{c} / \sigma>z \mid S\right\} & =z P_{\mathrm{R}} \\
P_{V}\left\{\eta_{c} / \sigma>z \mid V\right\} & =\left(z^{2}-1\right) P_{\mathrm{R}},
\end{aligned}
$$

where $P_{\mathrm{R}}$ is the Rayleigh distribution [(5)]. For large thresholds $(z \gg 1)$, the probability that the global surface maximum $\eta_{\max }$ exceeds $z$ over the space-time volume is expressed in FM as

$$
\begin{aligned}
& P_{\mathrm{FM}, \max }\left\{\eta_{\max } / \sigma>z \mid\left(N_{V}, N_{S}, N_{B}\right)\right\} \\
& \quad \approx\left[1-\left(1-P_{V}\right)^{N_{V}}\right]+\left[1-\left(1-P_{S}\right)^{N_{S}}\right]+\left[1-\left(1-P_{B}\right)^{N_{B}}\right] \\
& \quad \approx N_{V} P_{V}+N_{S} P_{S}+N_{B} P_{B} \approx\left(N_{V} z^{2}+N_{S} z+N_{B}\right) P_{\mathrm{R}} .
\end{aligned}
$$

The dimensionless most probable extreme value $\left(h_{\mathrm{FM}}=\right.$ $\eta_{\max } / \sigma$ ) expected to occur over the domain $X Y D$ is obtained as the value of $h$ that satisfies the implicit equation

$$
\left(N_{V} h^{2}+N_{S} h+N_{B}\right) \exp \left(-h^{2} / 2\right)=1 .
$$

The expected value of $\eta_{\max }$ is found via the asymptotic distribution of the largest values as

$$
E_{\mathrm{FM}, \max } / \sigma \approx h_{\mathrm{FM}}+\frac{\gamma}{h_{\mathrm{FM}}-\frac{2 N_{V} h_{\mathrm{FM}}+N_{S}}{N_{V} h_{\mathrm{FM}}^{2}+N_{S} h_{\mathrm{FM}}+N_{B}}},
$$


in agreement with Fedele (2012), where, however, the maxima were normalized by $H_{s}$.

Once the $x$ axis is aligned to the mean direction of wave propagation, the spectral mean wave and crest lengths are $L_{x}=13.6 \mathrm{~m}$ and $L_{y}=14.6 \mathrm{~m}$, which produce a short-crestedness parameter (Baxevani et al. 2003) $L_{x} / L_{y}=0.93$, characteristic of short-crested sea states. Moreover, the irregularity parameter $\alpha_{x t}=0.35$ indicates that the sea state is (on average) confused along the peak direction of propagation. The small value (0.004) of the parameter $\alpha_{y t}$ denotes that there is no organized and preferential wave movement along the $y$ axis. These conditions support large probabilities for extreme crests' occurrence, as visible in Fig. 9 where the FM results exceed the Rayleigh predictions of about $\sigma$. The expected maximum crest $E_{\mathrm{FM} \text {,max }} \approx 1.60 \mathrm{~m}$ (equivalent to $4.78 \sigma$ or $\left.1.19 H_{s}\right) \pm 0.10 \mathrm{~m}$ is still smaller than the observations.

Aiming at predicting the probabilities of the highest waves within the space-time ensemble, we have extended the FM model, including the secondorder contribution for each free wave mode (hereinafter FM2). In accordance with Fedele and Tayfun (2009), the dimensionless largest amplitude of the nonlinear stochastic wave group can be expressed in the Tayfun form given by (7). Following the study of Socquet-Juglard et al. (2005) that reports, in the context of the Piterbarg's theorem, a distribution for second-order space-time maximal crests (viz., the Piterbarg-Tayfun distribution), here the EDF of nonlinear extreme crest heights is derived [as done in Fedele et al. (2013) for spatial extremes] applying the transformation [(7)] in the FM model [(25)], namely,

$$
\begin{aligned}
& P_{\mathrm{FM} 2, \max }\left\{\eta_{\max } / \sigma>\xi \mid\left(N_{V}, N_{S}, N_{B}\right)\right\} \\
& \quad \approx\left(N_{V} z^{2}+N_{S} z+N_{B}\right) P_{\mathrm{R}} .
\end{aligned}
$$

The FM2 model provides the second-order expected value of the maximal crest over the domain $X Y D$ as

$$
\begin{aligned}
E_{\mathrm{FM} 2 \text { max }} / \sigma \approx\left(h_{\mathrm{FM}}+\frac{\mu}{2} h_{\mathrm{FM}}^{2}\right) & +\frac{\gamma}{h_{\mathrm{FM}}-\frac{2 N_{V} h_{\mathrm{FM}}+N_{S}}{N_{V} h_{\mathrm{FM}}^{2}+N_{S} h_{\mathrm{FM}}+N_{B}}}\left(1+\mu h_{\mathrm{FM}}\right),
\end{aligned}
$$

which is $1.83 \mathrm{~m}$ (equivalent to $5.46 \sigma$ or $1.37 H_{s}$ ) $\pm 0.13 \mathrm{~m}$ and well approximates the mean of the observed maxima; as well, the probability levels [(28)] match the EDF of the observed extremes (Fig. 9).

\section{c. Discussion}

Results presented in Fig. 9 deserve additional comments. The extreme value distributions derived from nonlinear $\mathrm{T}$ and TF models underestimate the empirical probabilities and the corresponding expected values. Nevertheless, each extreme is an independent realization of the maximal crest height in a series of $N \approx 500$ consecutive waves, and, in this respect, the time models should provide reliable probability levels. The differences may be explained by the fact that extremes have been recorded at specific spatial locations of the sea surface, that is, where wave groups maximal elevations have exceeded a specific threshold (i.e., $\eta_{i, \max }>1.25 H_{s}$ ).

Differently, the FM and FM2 models, which prescribe the probability of extremes for multidimensional random fields, provide a better approximation for the empirical data. In these models, the leading term of the functions (25) and (28) is the volume contribution $N_{V} z^{2}$ that keeps the probability at high levels by virtue of the number $N_{V}=1820$ and the term $z^{2}$ that multiply the Rayleigh distribution. Therefore, the space-time models use a larger number of waves compared to the time models. Along the same line, the number $N$ in the time models could be arbitrarily increased to derive higher probabilities levels, but in doing so the definition of $N$ (i.e., the average number of waves within a given duration) would lose its meaning. On the contrary, the number of waves adopted in FM and FM2 is consistent with the hypothesis underlying the theories for space-time maxima, and the inclusion of the irregularity parameters in (21) ensures the statistical independence between waves taken in time and space domain; in other words, the waves are not counted twice.

It is interesting to verify the sensitivity of the agreement between the experimental data and the FM2 predictions to the threshold used to select the highest waves of the space-time ensemble. To do so, we have modified the threshold toward values larger than $1.25 H_{s}$, thus reducing the extreme crest population size. In these cases too (not shown here), FM2 well reproduces the empirical exceedance probabilities for the entire range of observed maxima.

We note also that the FM and FM2 models provide exceedance probabilities larger than 1 for small thresholds (Fedele et al. 2013). To ensure probabilities smaller than 1 for all thresholds, a sound approximation is the Gumbel asymptotic limit of the space-time distributions given by 


$$
\begin{aligned}
& P_{\mathrm{FM}, \text { max }}\left\{\eta_{\mathrm{max}} / \sigma>z \mid\left(N_{V}, N_{S}, N_{B}\right)\right\} \approx \exp \left\{-\exp \left[-\left(z-h_{\mathrm{FM}}\right)\left(h_{\mathrm{FM}}-\frac{2 N_{V} h_{\mathrm{FM}}+N_{S}}{N_{V} h_{\mathrm{FM}}^{2}+N_{S} h_{\mathrm{FM}}+N_{B}}\right)\right]\right\}, \quad \text { and } \quad(30) \\
& P_{\mathrm{FM} 2 \text {,max }}\left\{\eta_{\mathrm{max}} / \sigma>\xi \mid\left(N_{V}, N_{S}, N_{B}\right)\right\} \approx \exp \left\{-\exp \left[-\frac{\left(\xi-h_{\mathrm{FM}}-\frac{\mu}{2} h_{\mathrm{FM}}^{2}\right)\left(h_{\mathrm{FM}}-\frac{2 N_{V} h_{\mathrm{FM}}+N_{S}}{N_{V} h_{\mathrm{FM}}^{2}+N_{S} h_{\mathrm{FM}}+N_{B}}\right)}{1+\mu h_{\mathrm{FM}}}\right]\right\}
\end{aligned}
$$

which well fit the exact probabilities (see FM2-GL in Fig. 9).

To strengthen the results presented, we have adopted a different strategy to capture the extremes of the spacetime ensemble. Following Fedele et al. (2013), who used different portions of the sea surface to demonstrate the dependence of the crest height upon the observed area, we have divided the sea surface framed by the stereo system into subsets spanning approximately $A / 4=$ $723 \mathrm{~m}^{2}$ of the whole surface. The $x-y$ plane shown in Fig. 3 has therefore been divided into four quadrants, each bounded by two axes, corresponding to the lines $x=0.0 \mathrm{~m}$ and $y=-46.4 \mathrm{~m}$. The same approach has been repeated along the time axis to split the entire sequence into four nonoverlapping subrecords approximately $450 \mathrm{~s}$ long. For each subrecord, the average number of waves is $N=125$. As a consequence, the entire spacetime ensemble has been divided into 16 space-time subensembles $\left(\mathrm{SE}_{j} ; j=1,2, \ldots, 16\right)$ whose spatial sizes $X$ and $Y$ used in the FM and FM2 models have been chosen approximating each subset with a square of equivalent area and sides $X=Y=26.9 \mathrm{~m}$. Within each $\mathrm{SE}_{j}$ the maximal surface elevation $\max \left[\eta\left(\mathrm{SE}_{j}\right)\right]$ has been collected and considered a member of the extreme crest population. Wave extreme positions have been checked to prevent maxima being taken on the same wave crest. Results of this analysis, shown in Fig. 10, confirm that the FM2 model well predicts the probabilities of maximal wave crests.

Additionally, we have gathered the maximum crest height $\eta_{0, \max }=\max \left[\eta\left(x_{0}, y_{0}, t\right)\right]$ at each fixed position $\left(x_{0}, y_{0}\right)$ of the sea surface elevation map shown in Fig. 3. The ensemble average over 72325 data is $\left\langle\eta_{0, \max }\right\rangle=$ $1.46 \mathrm{~m}$, in line with the predictions of the $\mathrm{T}$ and $\mathrm{TF}$ models for wave crest maxima. This agreement is not surprising and states that the theoretical models $\mathrm{T}$ and TF used to describe nonlinear wave crest distributions over time records hold for most of the observational points, where it is likely that a pointlike instrument gathers the waves passing through. There are, however, some specific fixed positions, those of records $B_{i}$, where the crest height maxima are outliers of the aforementioned models and yet are well described by the space-time distributions.

\section{Conclusions}

In this study, we have analyzed a space-time ensemble of sea surface elevations $\eta(x, y, t)$ in search of the largest crest heights. Wave data were acquired using a stereo camera system mounted on a fixed oceanographic platform and processed to produce a sequence of $3 \mathrm{D}$ elevation maps of the sea surface during a mild $\left(H_{s}=1.34 \mathrm{~m}, T_{p}=\right.$ $5.4 \mathrm{~s}$, and steepness of 0.06$)$ and mature $\left(c_{p} / U_{10}=0.76\right)$ sea state. From the ensemble $\eta(x, y, t)$ we have estimated skewness and kurtosis coefficients equal to 0.16 and 3.22 , respectively.

Within the space-time ensemble, we have isolated the $3 \mathrm{D}$ wave groups and selected 23 independent wave crests with height $\eta_{c}$ exceeding the rogue wave threshold for crest heights (i.e., $\eta_{c}>1.25 H_{s}$ ). Analysis of the time records at the positions $\left(x_{i}, y_{i}\right)$ of these maximal crests revealed that the highest elevations have an occurrence in excess of that derived by the standard statistics. This is true even when nonlinearities (for which the departure from Gaussian statistics was expressed as function of skewness and kurtosis) are taken into account, raising the issue of which probability function might be used to fit these extreme data.

Distribution of the extreme crests of the wave records has showed that the statistical models holding for timevarying nonlinear sea surface systems (viz., the Tayfun and Tayfun-Fedele models) underestimate the empirical maxima. In other words, there are points on the sea surface where extreme elevations may occur and for which all the standard distributions fail to describe their probability. More precisely, we claim that the apparent low probability of extreme elevations given by standard models may not be related to the rarity of such events but rather to the narrow spatial localization of high crests that reduces the probability of observing such phenomena if no preconditioning on the sampling point is introduced. This is possible only if space-time wave 


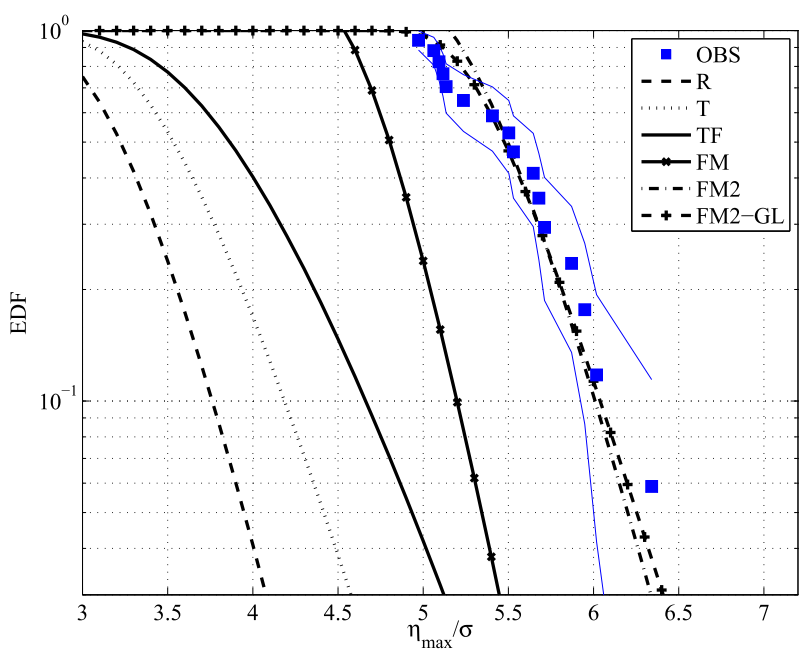

FIG. 10. Probability of exceedance (EDF) of dimensionless observed extreme crest heights $\max \left[\eta\left(\mathrm{SE}_{j}\right)\right]$ of the space-time subensembles $\mathrm{SE}_{j}(\mathrm{OBS})$. The empirical EDF stability band is plotted as blue solid line. Reference distributions of extremes: Rayleigh (R); Tayfun (T); Tayfun-Fedele (TF); Fedele (FM); second-order FM (FM2); and asymptotic Gumbel limit of FM2 (FM2-GL). The reference duration is $D=450 \mathrm{~s}$. Space-time extremes are computed over an area $X Y=26.9 \times 26.9 \mathrm{~m}^{2}=723 \mathrm{~m}^{2}$.

data are available. Indeed, the Tayfun and TayfunFedele models well perform for most of the observational points, whereas the highest crests' distribution derived from the records is well fitted by a second-order correction of the Fedele (2012) model for space-time maxima, which occur where the spatial wave groups are close to the apex of their development.

Therefore, even if the sea state analyzed was not extreme in terms of $H_{s}$, our results suggest that, if observational points are suitably chosen within a sample space, large waves are more numerous than expected by the standard time statistics. This should be a remarkable consideration for short-crested wave conditions, typical of stormy seas.

Acknowledgments. The authors gratefully acknowledge the funding from the Flagship Project RITMAREThe Italian Research for the Sea-coordinated by the Italian National Research Council and funded by the Italian Ministry of Education, University and Research within the National Research Program 2011-15. Authors wish to thank Prof. Mike Banner and Dr. Luigi "Gigi" Cavaleri for their helpful comments.

\section{REFERENCES}

Adler, R. J., 1981: The Geometry of Random Fields. John Wiley, $302 \mathrm{pp}$.

, and J. E. Taylor, 2007: Random Fields and Geometry. Springer, 448 pp.
Albarelli, A., E. Rodolà, and A. Torsello, 2010: Robust Camera calibration using inaccurate targets. Proc. 21st British Machine Vision Conf. 2010, Aberystwyth, Wales, British Machine Vision Association, 16.1-16.10.

Banner, M. L., X. Barthelemy, F. Fedele, M. Allis, A. Benetazzo, F. Dias, and W. L. Peirson, 2014: Linking reduced breaking crest speeds to unsteady nonlinear water wave group behavior. Phys. Rev. Lett., 112, 114502, doi:10.1103/PhysRevLett.112.114502.

Barbariol, F., A. Benetazzo, F. Bergamasco, S. Carniel, and M. Sclavo, 2014: Stochastic space-time extremes of wind sea states: Validation and modeling. Proc. 33rd Int. Conf. on Ocean, Offshore and Arctic Engineering, Paper OMAE201423997, San Francisco, CA, ASME Ocean, Offshore and Arctic Engineering Division, 11 pp., doi:10.1115/OMAE2014-23997.

Baxevani, A., and I. Rychlik, 2006: Maxima for Gaussian seas. Ocean Eng., 33, 895-911, doi:10.1016/j.oceaneng.2005.06.006.

—, K. Podgórski, and I. Rychlik, 2003: Velocities for moving random surfaces. Probab. Eng. Mech., 18, 251-271, doi:10.1016/ S0266-8920(03)00029-8.

Benetazzo, A., 2006: Measurements of short water waves using stereo matched image sequences. Coastal Eng., 53, 1013-1032, doi:10.1016/j.coastaleng.2006.06.012.

— shore stereo measurements of gravity waves. Coastal Eng., 64, 127-138, doi:10.1016/j.coastaleng.2012.01.007.

— S. Carniel, M. Sclavo, and A. Bergamasco, 2013: Wave-current interaction: Effect on the wave field in a semi-enclosed basin. Ocean Modell., 70, 152-165, doi:10.1016/j.ocemod.2012.12.009.

—, F. Bergamasco, F. Barbariol, A. Torsello, S. Carniel, and M. Sclavo, 2014: Towards an operational stereo system for directional wave measurements from moving platforms. Proc. 33rd Int. Conf. on Ocean, Offshore and Arctic Engineering, Paper OMAE2014-24024, San Francisco, CA, ASME Ocean, Offshore and Arctic Engineering Division, doi:10.1115/OMAE2014-24024.

Benjamin, T. B., and J. E. Feir, 1967: The disintegration of wave trains on deep water Part 1. Theory. J. Fluid Mech., 27, 417430, doi:10.1017/S002211206700045X.

Boccotti, P., 2000: Wave Mechanics for Ocean Engineering. Elsevier, 524 pp.

Bradski, G., and A. Kaehler, 2008: Learning OpenCV: Computer Vision with the OpenCV Library. 1st ed. O'Reilly Media, 555 pp.

Cartwright, D. E., and M. S. Longuet-Higgins, 1956: The statistical distribution of the maxima of a random function. Proc. Roy. Soc. London, A237, 212-232, doi:10.1098/rspa.1956.0173.

Cavaleri, L., 1999: The oceanographic tower Acqua Alta: More than a quarter of century activity. Nuovo Cimento Soc. Ital. Fis., 1, 1-112.

Dysthe, K., H. E. Krogstad, and P. Müller, 2008: Oceanic rogue waves. Annu. Rev. Fluid Mech., 40, 287-310, doi:10.1146/ annurev.fluid.40.111406.102203.

Fedele, F., 2008: Rogue waves in oceanic turbulence. Physica D, 237, 2127-2131, doi:10.1016/j.physd.2008.01.022. , 2012: Space-time extremes in short-crested storm seas. J. Phys. Oceanogr., 42, 1601-1615, doi:10.1175/JPO-D-11-0179.1.

—_, and M. A. Tayfun, 2009: On nonlinear wave groups and crest statistics. J. Fluid Mech., 620, 221-239, doi:10.1017/ S0022112008004424.

— , G. Gallego, A. Yezzi, A. Benetazzo, L. Cavaleri, M. Sclavo, and M. Bastianini, 2012: Euler characteristics of oceanic sea states. Math. Comput. Simul., 82, 1102-1111, doi:10.1016/ j.matcom.2011.05.009.

_ A. Benetazzo, G. Gallego, P.-C. Shih, A. Yezzi, F. Barbariol, and F. Ardhuin, 2013: Space-time measurements of oceanic sea states. Ocean Modell., 70, 103-115, doi:10.1016/j.ocemod.2013.01.001. 
Forristall, G. Z., 1978: On the statistical distribution of wave heights in a storm. J. Geophys. Res., 83, 2353-2358, doi:10.1029/ JC083iC05p02353.

- 2006: Maximum wave heights over an area and the air gap problem. Proc. 25th Int. Conf. on Offshore Mechanics and Arctic Engineering, Paper OMAE2006-92022, Hamburg, Germany, ASME, 11-15, doi:10.1115/OMAE2006-92022.

_ 2011: Maximum crest heights under a model TLP deck. Proc. 30th Int. Conf. on Ocean, Offshore and Arctic Engineering, Paper OMAE2011-49837, Rotterdam, Netherlands, ASME, 571-577, doi:10.1115/OMAE2011-49837.

Gallego, G., A. Yezzi, F. Fedele, and A. Benetazzo, 2011: A variational stereo method for the three-dimensional reconstruction of ocean waves. IEEE Trans. Geosci. Remote Sens., 49, 4445-4457, doi:10.1109/TGRS.2011.2150230.

Gumbel, E. J., 1958: Statistics of Extremes. Columbia University Press, $375 \mathrm{pp}$.

Hashimoto, N., T. Nagai, and T. Asai, 1994: Extension of the maximum entropy principle method for directional wave spectrum estimation. Proc. 24th Int. Conf. on Coastal Engineering, Kobe, Japan, ASCE, 232-246.

Haver, S., 2004: A possible freak wave event measured at the Draupner Jacket January 1 1995. Proc. Rogue Waves, Brest, France, IFREMER, 1-8. [Available online at http://www.ifremer.fr/ web-com/stw2004/rw/fullpapers/walk_on_haver.pdf.]

Hirschmüller, H., 2008: Stereo processing by semiglobal matching and mutual information. IEEE Trans. Pattern Anal. Mach. Intell., 30, 328-341, doi:10.1109/TPAMI.2007.1166.

Holthuijsen, L. H., 2008: Waves in Oceanic and Coastal Waters. Cambridge University Press, 387 pp.

Hwang, P. A., D. W. Wang, E. J. Walsh, W. B. Krabill, and R. N. Swift, 2000a: Airborne measurements of the wavenumber spectra of ocean surface waves. Part I: Spectral slope and dimensionless spectral coefficient. J. Phys. Oceanogr., 30, 2753-2767, doi:10.1175/1520-0485(2001)031<2753:AMOTWS > 2.0.CO;2.

,,,,---- and $-2000 \mathrm{~b}$ : Airborne measurements of the wavenumber spectra of ocean surface waves. Part II : Directional distribution. J. Phys. Oceanogr., 30, 2768-2787, doi:10.1175/1520-0485(2001)031<2768:AMOTWS > 2.0.CO;2.

Janssen, P. A. E. M., 2003: Nonlinear four-wave interactions and freak waves. J. Phys. Oceanogr., 33, 863-884, doi:10.1175/ 1520-0485(2003)33<863:NFIAFW $>2.0 . C O ; 2$.

Kharif, C., E. Pelinovsky, and A. Slunyaev, 2009: Rogue Waves in the Ocean. Springer Verlag, $216 \mathrm{pp}$.

Kosnik, M. V, and V. A. Dulov, 2011: Extraction of short wind wave spectra from stereo images of the sea surface. Meas. Sci. Technol., 22, 015504, doi:10.1088/0957-0233/22/1/015504.

Krogstad, H. E., and S. F. Barstow, 2000: A unified approach to extreme value analysis of ocean waves. Proc. 10th Int. Offshore Polar Engineering Conf., Seattle, WA, International Society of Offshore and Polar Engineers, 103-108.

_ J. Jiu, H. Socquet-Juglard, K. Dysthe, and K. Trulsen, 2004: Spatial extreme value analysis of nonlinear simulations of random surface waves. Proc. 23rd Int. Conf. on Offshore Mechanics and Arctic Engineering, Paper OMAE2004-51336, Vancouver, BC, Canada, ASME Ocean, Offshore, and Arctic Engineering Division, 285-295, doi:10.1115/OMAE2004-51336.

Kuik, A. J., G. P. Van Vledder, and L. H. Holthuijsen, 1988: A method for routine analysis of pitch and roll buoy wave data. J. Phys. Oceanogr., 18, 1020-1034, doi:10.1175/ 1520-0485(1988)018<1020:AMFTRA > 2.0.CO;2.

Leckler, F., F. Ardhuin, C. Peureux, A. Benetazzo, F. Bergamasco, and V. Dulov, 2015: Analysis and interpretation of frequency- wavenumber spectra of young wind waves. J. Phys. Oceanogr., doi:10.1175/JPO-D-14-0237.1, in press.

Lehner, S., and H. Gunther, 2004: Extreme wave statistics from radar data sets. Proc. 2004 Int. IEEE Geosci. Remote Sens. Symp., Anchorage, AK, IEEE, 1880-1883, doi:10.1109/IGARSS.2004.1370707.

Liu, P. C., 2013: Contemplating ocean wave measurements. Int. J. Geosci., 4, 229-233, doi:10.4236/ijg.2013.41A019.

Longuet-Higgins, M. S., 1952: On the statistical distribution of the heights of sea waves. J. Mar. Res., 11, 245-265.

- 1963: The effect of nonlinearities on statistical distribution in the theory of sea waves. J. Fluid Mech., 17, 459-480, doi:10.1017/ S0022112063001452.

_ 1975: On the joint distribution of the periods and amplitudes of sea waves. J. Geophys. Res., 80, 2688-2694, doi:10.1029/ JC080i018p02688.

Magnusson, A. K., and M. A. Donelan, 2013: The Andrea wave characteristics of a measured North Sea rogue wave. J. Offshore Mech. Arct. Eng., 135, 031108, doi:10.1115/1.4023800.

Mori, N., and P. E. M. Janssen, 2006: On kurtosis and occurrence probability of freak waves. J. Phys. Oceanogr., 36, 1471-1483, doi:10.1175/JPO2922.1.

Nieto Borge, J. C., 2004: Detection of ocean wave groupiness from spaceborne synthetic aperture radar. J. Geophys. Res., 109, C07005, doi:10.1029/2004JC002298.

—, K. Reichert, and K. Hessner, 2013: Detection of spatiotemporal wave grouping properties by using temporal sequences of X-band radar images of the sea surface. Ocean Modell., 61, 21-37, doi:10.1016/j.ocemod.2012.10.004.

Onorato, M., A. Osborne, M. Serio, and S. Bertone, 2001: Freak waves in random oceanic sea states. Phys. Rev. Lett., 86, 58315834, doi:10.1103/PhysRevLett.86.5831.

— S. Residori, U. Bortolozzo, A. Montina, and F. T. Arecchi, 2013: Rogue waves and their generating mechanisms in different physical contexts. Phys. Rep., 528, 47-89, doi:10.1016/ j.physrep.2013.03.001.

Piterbarg, V. I., 1996: Asymptotic Methods in the Theory of Gaussian Processes and Fields. Trans. Math. Monogr., Vol. 148, Amer. Math. Soc., 206 pp.

Romero, L., and W. K. Melville, 2010: Airborne observations of fetch-limited waves in the Gulf of Tehuantepec. J. Phys. Oceanogr., 40, 441-465, doi:10.1175/2009JPO4127.1.

fetch-limited conditions. J. Phys. Oceanogr., 41, 18211841, doi:10.1175/2011JPO4535.1.

Rosenthal, W., and S. Lehner, 2008: Rogue waves: Results of the MaxWave project. J. Offshore Mech. Arct. Eng., 130, 021006, doi:10.1115/1.2918126.

Socquet-Juglard, H., K. Dysthe, K. Trulsen, H. E. Krogstad, and J. Liu, 2005: Probability distributions of surface gravity waves during spectral changes. J. Fluid Mech., 542, 195-216, doi:10.1017/S0022112005006312.

Tayfun, M. A., 1980: Narrow-band nonlinear sea waves. J. Geophys. Res., 85, 1548-1552, doi:10.1029/JC085iC03p01548.

, 1981: Distribution of crest-to-trough wave heights. J. Waterw. Port Coastal Ocean Div., 107, 149-158.

— , and F. Fedele, 2007: Wave-height distributions and nonlinear effects. Ocean Eng., 34, 1631-1649, doi:10.1016/ j.oceaneng.2006.11.006

Wanek, J. M., and C. H. Wu, 2006: Automated trinocular stereo imaging system for three-dimensional surface wave measurements. Ocean Eng., 33, 723-747, doi:10.1016/j.oceaneng.2005.05.006.

Worsley, K. J., 1996: The geometry of random images. Chance, 9, $27-40$. 